
\title{
25 Research Square \\ Olfactory Disorder is the Specific Symptom to Distinguish Eosinophilic from non-eosinophilic Nasal Polyps
}

\section{Xiaodan Pan}

Beijing Tongren Hospital

\section{Yuan Zhang}

Beijing Tongren Hospital

Chengshuo Wang

Beijing Tongren Hospital

Luo Zhang ( $\nabla$ dr.luozhang@139.com )

Beijing Tongren Hospital https://orcid.org/0000-0002-0910-9884

\section{Research}

Keywords: Chronic rhinosinusitis, Nasal polyps, Olfactory disorder, Eosinophil

Posted Date: August 19th, 2020

DOI: https://doi.org/10.21203/rs.3.rs-60539/v1

License: (c) (i) This work is licensed under a Creative Commons Attribution 4.0 International License. Read Full License 


\section{Abstract}

Background: Patients with eosinophilic chronic rhinosinusitis with nasal polyps (eCRSwNP) have poorer outcomes after endoscopic sinus surgery and a higher recurrence rate. This study aimed to investigate the profile of clinical symptoms of eCRSWNP and the related risk factors.

Methods: We prospectively enrolled 298 CRSwNP inpatients from February 2019 to December 2019. Patients were divided into eCRSwNP and non-eCRSwNP groups based on the percentage of blood eosinophils. Clinical data on questionnaires, visual analogue scale (VAS) scores, and laboratory tests were collected. The differences in clinical symptoms, including nasal congestion, rhinorrhea, olfactory disorders, and head/facial pain, between the two groups were analyzed to identify the influential factors. Logistic analysis and receiver operating characteristic curves were used to determine the diagnostic benefit for the specific symptom in eCRSwNP patients.

Results: Of the four major clinical symptoms, nasal congestion and olfactory disorders were significantly different between eCRSwNP and non-eCRSwNP groups. Patients with eCRSwNP more frequently complained about olfactory disorders $(P=0.002)$, while patients with non-eCRSwNP mostly had nasal congestion $(P=0.001)$. The logistic analysis showed that the primary risk factors for olfactory disorders of eCRSwNP were disease duration $(P=0.014)$ and alcohol intake $(P=0.012)$. Olfactory disorders were not associated with the disease course of eCRSwNP but were correlated with the disease duration of noneCRSwNP $(P=0.008)$. When the clinical duration was less than 10 years, there was a significant difference in olfactory disorders between eCRSwNP and non-eCRSwNP groups $(P<0.01)$. However, when the clinical duration was over 10 years, the difference was not statistically significant $(P=0.264)$. The VAS score of olfactory disorders of over 5.75 could be used to predict the diagnosis of eCRSWNP (area under the curve $₫ 0.674,95 \%$ confidence intervals: $0.559-0.689, P=0.000$ ).

Conclusions: Olfactory disorder was the major nasal symptom that could be used to distinguish eCRSWNP and non-eCRSwNP. ECRSWNP patients were more prone to have olfactory dysfunction. Our findings suggested that evaluation of nasal symptoms would help diagnose eCRSwNP and determine subsequent clinical treatment strategies.

\section{Background}

Chronic rhinosinusitis (CRS), which referred to a chronic inflammatory disease of the nasal cavity and sinuses over 3 months, can cause various clinical symptoms such as nasal congestion and olfactory disorders (1). It was usually divided into two subtypes: CRS with nasal polyps (CRSwNP) and CRS without nasal polyps. Generally, CRSwNP requires surgical intervention and was known to have two subtypes, eosinophilic CRSwNP (eCRSwNP) and non-eosinophilic CRSwNP (non-eCRSwNP) (2); eCRSwNP was characterized by polarization of T helper 2 and expression of interleukin (IL)-4, IL-5, and IL$13(3,4)$, and non-eCRSwNP was characterized by IL-1, Interferon-gamma, insulin-like growth factor (IGF)-1, IGF-10, and IL-17-mediated cluster of differentiation 8 inflammation. They had overlapped clinical 
symptoms; for example, they both present with nasal congestion, olfactory disorders, rhinorrhea, and head or facial pain. In addition, they had different treatment responses and prognosis for drug or surgical intervention $(5,6)$. Therefore, the first aptitudinal diagnostic impression for these two subtypes was valuable for the further guidance of examination and treatment. To date, studies on diagnostic identification of eCRSwNP and non-eCRSwNP had been very fruitful. For example, those with peripheral blood eosinophil counts $\geq 0.215 \times 109 / \mathrm{L}$ or eosinophil percent $\geq 3.05 \%$ were classified as having eCRSwNP (7); differential diagnosis can be made through radiologic features: when the cutoff ratio of the ethmoid sinus score to the maxillary sinus score was over 2.59, the diagnosis of eCRSwNP was highly expected (8). However, the above predictive diagnoses of eosinophilic CRSWNP were associated with economic burden and time lag. Therefore, this study aimed to identify typical clinical symptoms that were meaningful for the differential diagnosis of eCRSWNP and non-eCRSWNP. The present findings could be used to guide the effective personalized treatment, thereby shortening the treatment time and improving the prognosis.

\section{Methods}

\section{Participants and study design}

We retrospectively analyzed clinical data of adult patients (age $\geq 18$ years) who met the diagnosis of CRSwNP. The diagnosis of CRSwNP was based on the standard criteria of the European Position Paper on Rhinosinusitis and Nasal Polyps guidelines (1). All the enrolled patients were hospitalized and underwent endoscopic sinus surgery at Beijing Tongren hospital from February 2019 to December 2019. Among 500 invited adult CRSwNP patients, 144 patients with the diagnosis of fungal sinusitis or CRS without nasal polyps were excluded, finally 298 were enrolled in this study. The data of clinical characteristics, chief complaints, laboratory tests, nasal functions, and endoscopic and radiologic scores were collected. The visual analogue scale (VAS) questionnaire with the accuracy of 0.1 was completed by participants, and the items included various clinical symptoms, such as nasal congestion, rhinorrhea, olfactory disorders, and head/facial pain. Nasal congestion was given as a sense of congestion unilaterally or bilaterally. Rhinorrhea was asked as a condition where the nasal cavity was filled with a significant amount of mucus fluid. Olfactory disorders was described as the inability to perceive odor or loss of the sense of smell. Head /facial pain was asked either the forehead or the maxillary portion suffering a sense of fullness and pain. Among the 298 patients, 12 did not complete the questionnaires; therefore, 286 questionnaires were obtained and analyzed. We grouped the final 298 CRSwNP patients into two subgroups, eosinophilic and non-eosinophilic CRSWNP groups. The blood eosinophil percentage higher than 3.05\% was set as a cutoff value for distinguishing eosinophilic and non-eosinophilic NP (7). Clinical characteristics of eosinophilic and non-eosinophilic NP, especially nasal congestion, rhinorrhea, olfactory disorders, and head/facial pain, were compared. We also carried out a stratified analysis to further determine the symptomatic difference based on the clinical duration. This study was approved by 
the Ethics Committee of Beijing Tongren Hospital, and written informed consent was obtained from patients before data collection.

\section{Clinical data collection}

Collected data included basic clinical information, chief complaints, laboratory tests, allergy status, serum total lgE, nasal functions, and endoscopic and radiologic scores. The basic clinical information included age, gender, disease duration, previous surgery, smoke, alcohol, and asthma history. The laboratory tests included blood neutrophil percent, blood lymphocyte percent, blood monocyte percent, and blood eosinophil percent. The blood samples were taken before surgery and were analyzed by an automated analyzer. For NP patients, the duration of disease and history of prior nasal surgery or comorbidity with asthma were recorded. The evidence of nasal polyps was confirmed under endoscopy in the unilateral or bilateral nasal cavity. Nasal resistance was measured by HRR2 four-phase rhinomanometry (RhinoLab GmbH, Rendsburg, Germany) in an examination room at a temperature of 22 ${ }^{\circ} \mathrm{C}$ to $24^{\circ} \mathrm{C}$ and $40 \%$ to $70 \%$ humidity. Nasal resistances were assessed at $75 \mathrm{~Pa}$ and $150 \mathrm{~Pa}$, respectively, and total nasal resistance and unilateral nasal resistance were recorded at each pressure (9). The serum total IgE (kU/I) was evaluated by Immuno-Cap Phadiatop (Pharmacia, Uppsala, Sweden). Pre-operatively, an endoscopic examination was scored according to the Lund-Kennedy system, and edema, nasal discharge, scarring, and crusting were assessed (10). Computed tomography (Philips Health Care, Best,

The Netherlands) of paranasal sinuses was performed for all patients, and scoring was conducted using the Lund-Mackay system (11): no soft tissue in the sinus, scored 0; partial soft tissue, scored 1; and full of tissue, scored 2. Ethmoid sinus scores $(E)$ were divided by mastoid scores $(M)$ to calculate $E / M$, in which $\mathrm{E}$ stood for the sum of anterior and posterior ethmoid sinus scores on both sides and $\mathrm{M}$ stood for the sum of bilateral maxillary sinus scores. Allergy status was confirmed based on Immuno-Cap Phadiatop (Pharmacia, Uppsala, Sweden) (cutoff $\geq 0.35 \mathrm{kU} / \mathrm{mL}$ ).

\section{Statistical analysis}

Data were analyzed using SPSS version 23.0 software (IBM Corp, Armonk, NY). All data were expressed as median and interquartile range or number and percentage. The Mann-Whitney $U$ test for unpaired comparisons was used for the comparison between groups. The relationship between chief complaints and clinical information was evaluated by relevance analysis and logistic regression analysis. Odds ratios $(\mathrm{OR})$ and $95 \%$ confidence intervals $(\mathrm{Cl})$ were calculated with the logistic regression model. The receiver operating characteristic (ROC) curve was used to distinguish eCRSWNP and non-eCRSWNP. An area under the curve (AUC) of $>0.9$ was defined as the higher predictive value, an AUC of 0.7-0.9 the moderate value, and an AUC of 0.5-0.6 the lower value. The optimal cutoff point was the one corresponding to the data with the highest sensitivity/specificity. $P$ values $<0.05$ were considered significant. 


\section{Results}

\section{Demographics of participants}

This study observed a total of 298 CRSwNP patients, including 152 eosinophilic NP patients (51\%) and 146 non-eosinophilic patients (49\%). The age was similar distributed (forty to fifty years) in both groups $(P=0.192)$, and the male/female ratio was nearly 2:1 in both groups. Patients had CRSwNP for quite different durations (from at least 3 months to at most 40 years). However, there were no significant differences in disease duration ( $P=0.496)$, as well as tobacco $(18.4 \%$ vs $20.5 \%, P=0.664)$ or alcohol $(8.55 \%$ vs $13.7 \%, P=0.158)$ intake, between the two groups. The eCRSwNP patients tended to undergo more endoscopic surgery in the past years, but the difference was not significant $(P=0.83)$. Notably, eCRSwNP patients were more likely to have history of asthma than non-eCRSwNP patients $(13.8 \% \mathrm{vs}$ $4.28 \%, P=0.004)$. In addition, non-eCRSwNP patients seemed to be weighed more than eCRSwNP patients $(P=0.048)$. Patients in both groups were in the allergy status $(28.83 \%$ vs $31.67 \%, P=0.700)$, and the difference was not significant between the two groups. Moreover, some laboratory tests revealed distinct differences between eCRSwNP and non-eCRSwNP groups. The P-value between the two groups for blood eosinophil percent, blood neutrophil percent, blood monocyte percent, and serum total IgE were $0.000,0.000,0.007$, and 0.002 , respectively. Furthermore, eCRSwNP patients had more eosinophils $(P=$ $0.000)$. The difference in the $E / M$ ratio between the two groups was significant $(P=0.000)$, but not significantly different in total Lund-Kennedy endoscope scores $(P=0.487)$ and Lund-Mackay CT scores $(P=0.219)$. The details of demographic and clinical characteristics were displayed in Table 1. 
Table 1

Clinical characteristics of participants.

\begin{tabular}{|c|c|c|c|}
\hline Variables & $\begin{array}{l}\text { ECRSwNP } \\
(N=152)\end{array}$ & $\begin{array}{l}\text { Non-eCRSwNP } \\
(\mathrm{N}=146)\end{array}$ & $P$ values \\
\hline Age (years), mean (SD) & $43.16 \pm 11.58$ & $44.88 \pm 14.71$ & 0.192 \\
\hline Sex (male/female), $\mathrm{n}$ & $94 / 58$ & $48 / 25$ & 0.483 \\
\hline Disease duration (years) & $5.67 \pm 6.06$ & $6.62 \pm 6.84$ & 0.469 \\
\hline Previous FESS surgery, n (\%) & $20(13.2 \%)$ & $9(12.3 \%)$ & 0.830 \\
\hline Smoker, n (\%) & $28(18.4 \%)$ & $15(20.5 \%)$ & 0.644 \\
\hline Drinker, n (\%) & $13(8.55 \%)$ & $10(13.7 \%)$ & 0.158 \\
\hline Asthma history (\%) & $21(13.8 \%)$ & $3(4.28 \%)$ & 0.004 \\
\hline Allergy status, $+/-\rrbracket \% \square$ & $32(28.83 \%)$ & 19 (31.67\%】 & 0.700 \\
\hline Blood eosinophil percent (\%) & $7.73 \pm 9.12$ & $1.73 \pm 0.80$ & 0.000 \\
\hline Blood neutrophil percent (\%) & $54.80 \pm 31.34$ & $59.83 \pm 9.22$ & 0.000 \\
\hline Blood monocyte percent (\%) & $6.99 \pm 4.27$ & $6.21 \pm 1.45$ & 0.007 \\
\hline Serum total lgE (kU/I) & $209.82 \pm 287.30$ & $199.04 \pm 499.99$ & 0.002 \\
\hline Blood lymphocyte percent (\%) & $32.93 \pm 7.34$ & $31.44 \pm 8.64$ & 0.070 \\
\hline Eosinophils exfoliated cells & $1.56 \pm 0.50$ & $1.81 \pm 0.40$ & 0.000 \\
\hline Endoscope score(total) & $6.82 \pm 2.46$ & $7.10 \pm 2.85$ & 0.487 \\
\hline Lund-Kennedy endoscopic score, E/M ratio* & $2.26 \pm 0.74$ & $1.87 \pm 0.96$ & 0.000 \\
\hline Lund-Mackay CT score (total) & $15.51 \pm 6.33$ & $14.31 \pm 6.56$ & 0.219 \\
\hline \multicolumn{4}{|c|}{$\begin{array}{l}\text { ECRSwNP: eosinophilic chronic rhinosinusitis with nasal polyps; SD: standard deviation; E/M: } \\
\text { ethmoid versus mastoid. }\end{array}$} \\
\hline \multicolumn{4}{|l|}{ Analysis method: Mann-Whitney U test } \\
\hline
\end{tabular}

\section{Comparison of profiles of nasal symptoms between eosinophilic and non-eosinophilic NP groups}

A total of 286 patients completed the questionnaires, including148 eCRSwNP patients and 138 noneCRSWNP patients. Olfactory disorder was the symptom with the most distinct difference between the 
two groups, and was the unique symptomatic complaint that was significantly different between the two groups $(P=0.000)$. In addition, the VAS scores of nasal congestion, rhinorrhea, olfactory disorder, and head/facial pain did not significantly differ between these two groups. We further evaluated nasal congestion through rhinomanometry and found that unilateral or bilateral nasal resistance at either 75pa or 150pa was not significantly different between the two groups (see Additional Table 1). Moreover, patients in the two groups complained about different primary symptoms. CRSwNPs patients were more likely to be puzzled by loss of smell $(P=0.002)$, and non-eCRSwNPs patients were more likely to be perplexed by nasal congestion $(P=0.001)$. The ratios of loss of smell were $57.4 \%$ and $39.1 \%$ in eCRSwNP and non-eCRSwNP groups, respectively. The ratios of nasal congestion were $38.5 \%$ and $58.0 \%$ in eosinophilic and non-eosinophilic NP groups, respectively. Details of the comparison of nasal symptoms were displayed in Table 2.

Table 2

Comparison of profiles of nasal symptoms between eCRSWNP and non-eCRSWNP patients.

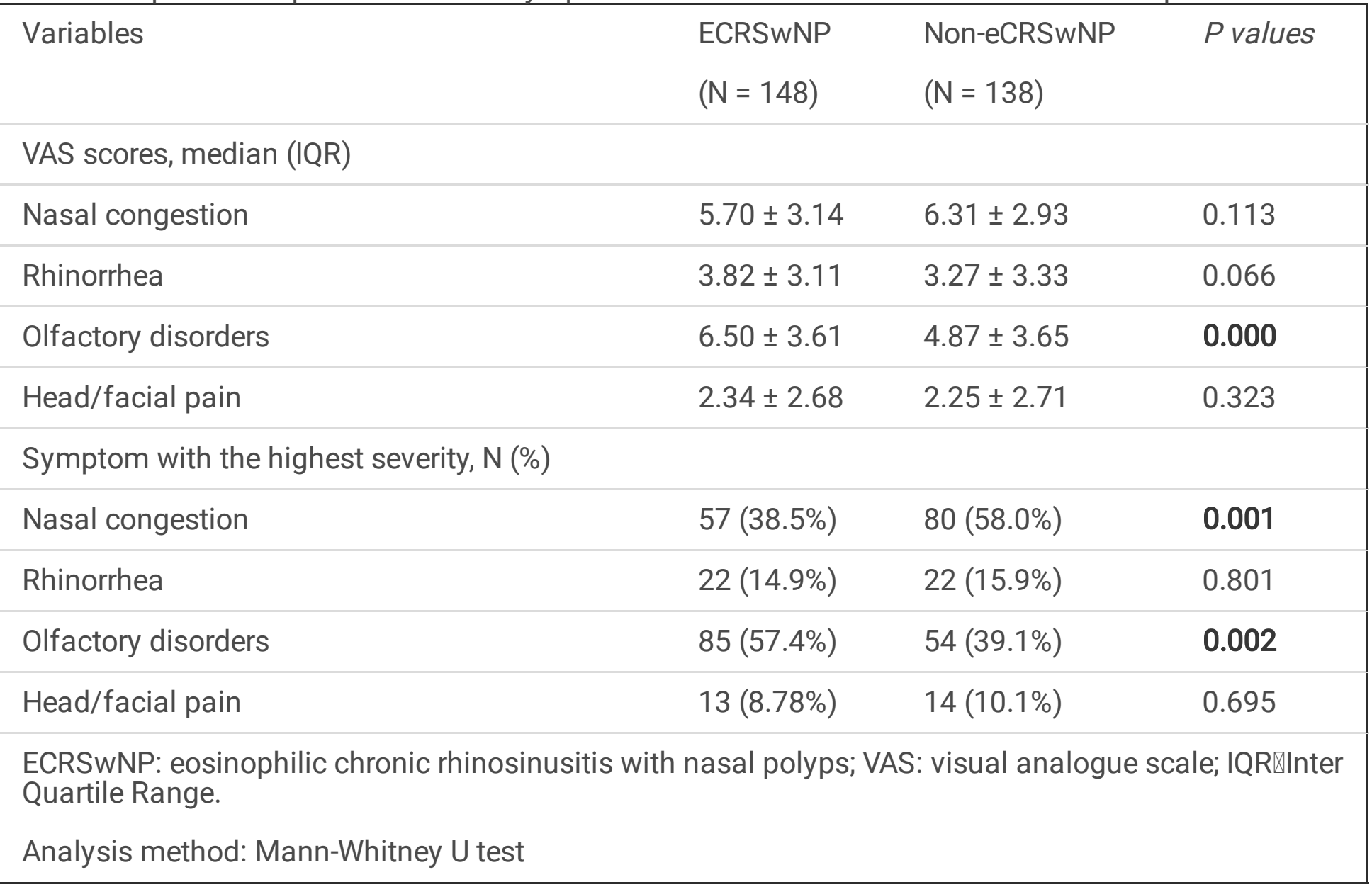

To further determine the factors associated with olfactory function in CRSwNP, relevance analysis and logistic regression analysis were conducted based on between-group comparison analysis. Relevance analysis revealed that smell loss was related to disease duration $(P=0.026)$, blood eosinophilic percent $(P=0.01)$ and eosinophilic absolute counts $(P=0.001)$ in overall CRSwNP patients and blood neutrophilic percent $(P=0.031)$, blood lymphocyte percent $(P=0.014)$, and blood neutrophilic absolute counts $(P=0.013)$ in eCRSwNP patients. In the non-eCRSwNP group, olfactory disorders were related to 
disease duration $(P=0.020)$ and alcohol intake $(P=0.018)$. Further, the multiple logistic regression found that both total CRSwNP and non-eCRSwNP were significantly correlated with disease duration $(P=0.014$, $\mathrm{OR}=11.23 ; P=0.008, \mathrm{OR}=1.21)$. These details were displayed in Table 3.

Table 3

Factors associated with olfactory function in CRWwNPs patients.

\begin{tabular}{|c|c|c|c|c|c|}
\hline \multirow[t]{2}{*}{ Variables } & \multicolumn{2}{|c|}{ Relevant analysis } & \multicolumn{3}{|c|}{ Logistic analysis } \\
\hline & Coefficient & $\begin{array}{l}P \\
\text { value }\end{array}$ & OR & $95 \% \mathrm{Cl}$ & $\begin{array}{l}P \\
\text { values }\end{array}$ \\
\hline \multicolumn{6}{|l|}{ Total CRSwNP } \\
\hline Disease duration & 0.132 & 0.026 & 1.23 & $0.69-2.20$ & 0.014 \\
\hline Blood Eosinophilic percent & 0.152 & 0.01 & 2.09 & $0.94-4.67$ & 0.001 \\
\hline Drinker & -0.197 & 0.011 & 6.63 & $\begin{array}{l}1.49- \\
29.66\end{array}$ & 0.012 \\
\hline \multicolumn{6}{|l|}{ ECRSwNP } \\
\hline Blood Neutrophilic percent $\varangle \% \bigotimes$ & 0.177 & 0.031 & 0.754 & $\begin{array}{l}0.179- \\
3.18\end{array}$ & 0.807 \\
\hline Blood Lymphocyte percent $₫ \% \bigotimes$ & -0.201 & 0.014 & 2.20 & $0.72-6.70$ & 0.228 \\
\hline $\begin{array}{l}\text { Blood Neutrophil absolute } \\
\operatorname{counts}\left(10^{\wedge} 9 \mathrm{~g} / \mathrm{L}\right)\end{array}$ & 0.205 & 0.013 & 1.48 & $0.35-6.37$ & 0.472 \\
\hline \multicolumn{6}{|l|}{ Non-eCRSwNP } \\
\hline Disease duration & 0.266 & 0.020 & 1.21 & $0.53-2.77$ & 0.008 \\
\hline Drinker & -0.201 & 0.018 & 7.34 & $\begin{array}{l}1.63- \\
33.06\end{array}$ & 0.009 \\
\hline \multicolumn{6}{|c|}{ CRSwNP: chronic rhinosinusitis with nasal polyps; OR: Odds ratio; Cl: Confidence interval. } \\
\hline Analysis method: Mann-Whitne & & & & & \\
\hline
\end{tabular}

By using the ROC curves, we further explored the prominent predictive symptoms that could be used to differentiate eCRSWNP and non-eCRSwNP. Among the four symptoms, olfactory dysfunction was the only valuable clinical marker for the predictive diagnosis of eCRSwNP. The details are displayed in Table 4 , and the ROC curves are shown in Figure 1. The AUC curves of four symptoms. The AUC of olfactory disorder (AUC $=0.642$ ) was higher than those of other symptoms, with a sensitivity of 0.662 and specificity of $0.594(P=0.000, \mathrm{Cl} 0.559-0.689)$. 
Table 4

Diagnostic predictive values of parameters.

\begin{tabular}{|lllllll|}
\hline Parameters & AUC & $95 \% \mathrm{Cl}$ & Cutoff value & Sensitivity & Specificity & $P$ values \\
\hline Olfactory disorders & 0.624 & $0.559-0.689$ & 5,75 & 0.662 & 0.594 & 0.000 \\
\hline Head/facial pain & 0.563 & & & & 0.067 \\
\hline Rhinorrhea & 0.534 & & & 0.327 \\
\hline Nasal congestion & 0.446 & & & 0.113 \\
\hline AUC: area under the curve; Cl: Confidence interval. & & & \\
\hline
\end{tabular}

In order to further examine the association of olfactory dysfunction with disease duration in eCRSwNP and non-eCRSwNP, we classified the patients into the following four groups based on the disease duration: less than 1 year, 1 to 5 years, 6 to 10 years, and more than 10 years. The results revealed significant differences between eCRSwNP and non-eCRSwNP patients in the three subgroups with disease duration of less than 10 years $(P=0.013, P=0.001, P=0.011)$. However, the difference between the two groups with the over ten-year duration was not significant $(P=0.264)$. Moreover, blood eosinophilic percent $(P=0.001, \mathrm{OR}=2.09)$ and eosinophilic absolute counts $(P=0.001, \mathrm{OR}=2.63)$ were correlated with loss of smell in the overall CRSwNP group. In addition, alcohol intake $(P=0.009, \mathrm{OR}=$ 7.34) was another apparent influential factor for olfactory dysfunction in non-eCRSwNP patients. The details were displayed in Table 5. 
Table 5

Comparison of nasal symptoms between eCRSwNP and non-eCRSwNP patients based on disease duration.

\begin{tabular}{|llll|}
\hline Variables & eCRSwNP & Non-eCRSwNP & P values \\
VAS scores, median (IQR) & $(\mathrm{N}=148)$ & $(\mathrm{N}=138)$ & \\
\hline Duration: $<1$ year & $(\mathrm{N}=26)$ & $(\mathrm{N}=30)$ & \\
\hline Nasal congestion & $4.50 \pm 2.97$ & $4.93 \pm 3.42$ & 0.633 \\
\hline Rhinorrhea & $3.11 \pm 2.83$ & $2.49 \pm 3.05$ & 0.268 \\
\hline Olfactory disorders & $6.07 \pm 3.67$ & $3.35 \pm 3.44$ & 0.013 \\
\hline Head/facial pain & $2.75 \pm 3.70$ & $1.47 \pm 1.96$ & 0.071 \\
\hline Duration: 1-5 year & $(\mathrm{N}=66)$ & $(\mathrm{N}=44)$ & \\
\hline Nasal congestion & $5.48 \pm 3.42$ & $6.24 \pm 2.98$ & 0.386 \\
\hline Rhinorrhea & $4.24 \pm 3.24$ & $3.17 \pm 2.80$ & 0.068 \\
\hline Olfactory disorders & $6.76 \pm 3.49$ & $5.18 \pm 3.35$ & 0.010 \\
\hline Head/facial pain & $2.40 \pm 2.63$ & $2.45 \pm 2.72$ & 0.864 \\
\hline Duration: 6-10 year & $(\mathrm{N}=42)$ & $(\mathrm{N}=44)$ & 0.546 \\
\hline Nasal congestion & $6.54 \pm 2.85$ & $6.91 \pm 2.35$ & 0.782 \\
\hline Rhinorrhea & $3.62 \pm 3.18$ & $3.79 \pm 3.81$ & 0.696 \\
\hline Olfactory disorders & $6.52 \pm 3.78$ & $4.46 \pm 3.57$ & 0.011 \\
\hline Head/facial pain & $2.47 \pm 2.87$ & $2.30 \pm 3.07$ & 0.266 \\
\hline Duration: > 10 year & $(\mathrm{N}=14)$ & $(\mathrm{N}=20)$ & \\
\hline Nasal congestion & $11.06 \pm 17.37$ & $7.19 \pm 2.54$ & 0.158 \\
\hline Rhinorrhea & $3.76 \pm 2.68$ & $3.54 \pm 3.61$ & \\
\hline Olfactory disorders & $12.40 \pm 25.47$ & $7.34 \pm 3.51$ & \\
\hline Head/facial pain & $2.22 \pm 3.15$ & $2.89 \pm 2.64$ & \\
\hline eCRSwNP: eosinophilic chronic rhinosinusitis with nasal polyps; $\mathrm{VAS}: \mathrm{visual}$ & & \\
\hline Quartile Ran. & & & \\
\hline
\end{tabular}

\section{Discussion}


Olfactory disorder was an important clinical manifestation of CRSwNP. European and American scholars have statistically shown that about $14 \%$ and $10 \%$ of CRS patients have progressive olfactory dysfunction, respectively $(1,12)$. Although olfactory disorder was not fatal, it could seriously lower the quality of life and result in loss of appetite, malnutrition, immunity dysfunction, and even food poisoning due to accidental consumption of spoiled food $(13,14)$. Studies had shown that the most common clinical symptom of eCRSwNP was olfactory disorder $(15,16)$, and ECRSwNP was usually resistant to drug therapy and surgical treatment and was prone to relapse after treatment. Therefore, eCRSwNP, an important category of refractory rhinosinusitis (17), had become the annoying bottleneck in the treatment of eosinophilic nasal polyps with functional endoscopic sinus surgery. To date, researchers had developed a variety of diagnostic approaches for eCRSwNP, such as blood routine tests, radiologic examinations, and tissue histopathology. Nevertheless, all these methods were invasive or need to wait for a long time to get the reports. Therefore, it was important to diagnose eCRSwNP by recognizing the first noticed primary complaints.

A total of 298 patients with CRSwNP were prospectively analyzed in our study, and the clinical characteristics of eCRSWNP and non-eCRSWNP were collected and described. Using the characteristics of the patients' clinical symptoms, we explored the initial diagnostic tendency. In our study, most CRSwNP patients were aged between forty and fifty years with a male dominance. Patients with eCRSwNP had a familiar history of asthma, and the findings were consistent with those in many previous studies (18-20). In addition, the CT manifestation of eCRSwNP was the centralization of polyps and edema mucosa in the ethmoid sinus and olfactory cleft region, while the non-eCRSwNP was predominantly observed in the maxillary sinus. Our findings were similar to those in previous studies, which showed that the ethmoid and the maxillary sinus were predominantly involved in eCRSwNPs and non-eCRSwNPs, respectively (8, $21,22)$. Notably, eCRSwNP patients complained mostly about olfactory dysfunction in the early days; non-eCRSwNP patients commonly presented with nasal congestion with olfactory sensation worsening over time. Therefore, olfactory dysfunction might be the most important clinical symptom in eCRSwNP and non-eCRSwNP patients.

Regarding physiology and pathophysiology of olfactory function, the first step in olfaction was to diffuse the odors to the olfactory epithelium located on the cribriform plate, the upper part of the nasal septum, and the middle and superior turbinate, which were called the olfactory area. Odors were dissolved in the mucus layer and then activated the olfactory receptors (14). Each odor can be recognized by an olfactory receptor through a complex code to help distinguish millions of odors. After processing, the olfactory information was integrated into the olfactory bulb and then transmitted to the limbic system (sentiment), to the hippocampus (memory), and finally to the olfactory cortex (23). Olfactory disorders were associated with complicated fusions of a variety of triggers and involved not only physical obstructions of odors conduction, especially in the olfactory area (24), but also immunologic and chemical effects. Polyps in eCRSwNP were usually bilaterally involved in lesions, mostly in the ethmoid sinus, ostiomeatal complex, and olfactory area, where the olfactory epithelium was richly distributed. The polyps and edema of mucosa mechanically blocked the transmission of odors to the olfactory area $(15,25)$. The immunologic effect of eCRSWNP mainly involved type 2 inflammation due to nasal polyps or edema and 
was associated with production of IL-3, IL-5, IL-13, granulocyte-macrophage colony-stimulating factor, and eotaxin and up-regulation of adhesion molecules, thereby leading to ecclasis and degeneration of the olfactory epithelium until falling-down of olfactory function $(26,27)$. Moreover, neurotoxic mediators, known as eosinophilic derived neurotoxins, which were released by eosinophils, can damage the olfactory nerve (28), impede the transmission of odors to the mucus and epithelium, and impair regeneration of neurons. As one of the four main proteins (major basic protein, eosinophil peroxidase, eosinophil-derived neurotoxin, and eosinophilic cationic protein [ECP]) in the eosinophils (29), ECP played a devastating role by activating infiltration of the eosinophilic inflammatory cells in the neuroepithelium. In vitro studies had found that ECP gradually increased after exposure to pollen, and the increase was associated with the progression of hyposmia. Nevertheless, ECP can slower the frequency of nasal ciliary movement, even in the epithelial cells (30). All these complicated factors made the sensory receptor fragile to the pathologic stimuli $(25,27)$.

In our study, patients with eCRSwNP had more severe olfactory disorders than those with non-eCRSwNP. The number and severity of symptoms were significantly higher in eCRSwNP patients than in noneCRSwNP patients. By multiple regression analysis, we found that the high eosinophilic level in the peripheral blood was an independent risk factor for olfactory dysfunction in CRSwNP patients. The higher the eosinophil level, the more severe the olfactory dysfunction would be. Haruna et al. reported that olfactory dysfunction was more significant when there were a large number of eosinophils in the ethmoid sinus mucosa than when there were a small number of eosinophils in the tissue (15). Mori et al. observed that eosinophilic CRS patients had more severe olfactory dysfunction than non-eosinophilic CRS patients, and smoking was a risk factor for olfactory dysfunction (31). Therefore, it was believed that patients with eCRSwNP were more likely to suffer from loss of smell, probably due to the high eosinophilic level. Furthermore, the ROC curves, with 5.75 as the cutoff value, revealed the significance of olfactory dysfunction in clinical symptoms. All above, if the VAS score of olfactory disorders was beyond 5.75 , the patient probably had eCRSwNP, and the symptomatic marker for olfactory dysfunction would be practical and instructive.

The analysis of the risk factors found that olfactory disorders were correlated with the eosinophilic levels, but not with the disease duration, in eCRSwNP patients. In contrast, olfactory disorders were related to the disease duration in non-eCRSwNP patients. The longer the duration, the more severe the hyposmia.

Further, stratified analysis of the disease duration showed that when the disease duration was less than 10 years, patients with eCRSWNP had more apparent olfactory disorders, while patients with noneCRSwNP mainly had nasal congestion. However, when the disease duration was more than 10 years, the symptoms of olfactory disorders became confusing in both groups. These findings indicated that with the progression of the disease, olfactory dysfunction became more significant in non-eCRSWNP patients, probably because the reduplicate stimulation destroyed the epithelium in the context of chronic inflammation $(28,30)$.

There were some limitations in the present study. First, the sample size was limited so that the disease duration could not be further subdivided, thus probably decreasing the accuracy of the predictive 
analysis. Further studies with a large sample size were needed to identify an accurate time boundary for better distinguishing between eCRSWNP and non-eCRSwNP. Second, the subjective judgment of VAS scores was used for the diagnosis of olfactory disorder; thus, other objective methods (such as the Sniffin' Sticks test) should be applied to increase the accuracy of diagnosis in future studies. Third, the diagnostic criteria used in this study were based on the blood eosinophilia and were mainly used to distinguish eosinophilia-dominant from non-eosinophilia-dominant. Therefore, a relatively broad standard would be more appropriate to define the two types in clinical practice and to help diagnose the olfactory disorders.

\section{Conclusions}

Olfactory disorder was the major nasal symptom that could be used to distinguish eCRSWNP and noneCRSwNP. The accurate time boundary for better distinguishing between eCRSWNP and non-eCRSwNP would be our next step for further study. ECRSwNP patients were more prone to have olfactory dysfunction. Evaluation of nasal symptoms would help diagnose eCRSWNP and determine targeted subsequent clinical treatment strategies.

\section{List Of Abbreviations}

AUC: area under the curve; Cl: confidence intervals; CRS: chronic rhinosinusitis; ECP: eosinophilic cationic protein; eCRSwNP: eosinophilic chronic rhinosinusitis with nasal polyps; IGF: insulin-like growth factor; IL: interleukin; OR: Odds ratios; ROC: receiver operating characteristic; VAS: visual analogue scale.

\section{Declarations}

\section{- Ethics approval and consent to participate}

This study was approved by the Ethics Committee of Beijing Tongren Hospital, and written informed consent was obtained from patients before data collection.

\section{- Consent for publication}

Consent of publication was obtained from all subjects.

\section{- Availability of data and materials}

The datasets used and analyzed during the current study was available from the corresponding author on reasonable request

\section{- Competing interests}

All authors declare no financial or commercial conflicts of interest. 


\section{- Funding}

This work was supported by grants from the national key R\&D program of China (2016YFC0905200), the program for the Changjiang scholars and innovative research team (IRT13082), the national natural science foundation of China (81630023), Beijing municipal administration of hospitals' mission plan (SML20150203), and Beijing talents foundation (2018000021223ZK14).

\section{- Authors' contributions}

Yuan Zhang was the designer of the study. Yuan Zhang and Xiaodan Pan collected and analyzed the data, drafted and revised the manuscript. Chengshuo Wang and Luo Zhang revised the paper, supervise the process and approve the final submission.

\section{- Acknowledgements}

Thanks to all the clinical colleague that helped completed the questionnaire and support our study.

\section{- Authors' information}

Xiaodan Pan, chief resident of otolaryngology head and surgery of Beijing Tongren hospital. Yuan Zhang, associate chief doctor of otolaryngology head and surgery. Chengshuo Wang, vice director of department of rhinology. Luo Zhang, president of international allergy association and the dean of Beijing Tongren hospital.

\section{References}

1. Fokkens W, Desrosiers M, Harvey R, Hopkins C, Mullol J, Philpott C, et al. EPOS2020: development strategy and goals for the latest European Position Paper on Rhinosinusitis.

2. Tokunaga T, Sakashita M, Haruna T, Asaka D, Takeno S, Ikeda $\mathrm{H}$, et al. Novel scoring system and algorithm for classifying chronic rhinosinusitis: the JESREC Study. Allergy. 2015;70:995-1003.

3. Baba S, Kagoya R, Kondo K, Suzukawa M, Ohta K, Yamasoba T. T-cell phenotypes in chronic rhinosinusitis with nasal polyps in Japanese patients. Allergy Asthma Clin Immunol. 2015;11:33.

4. Otto BA, Wenzel SE. The role of cytokines in chronic rhinosinusitis with nasal polyps. Curr Opin Otolaryngol Head Neck Surg. 2008;16:270-4.

5. Van Crombruggen $K$, Zhang N, Gevaert P, Tomassen P, Bachert C. Pathogenesis of chronic rhinosinusitis: inflammation. J Allergy Clin Immunol. 2011;128:728-32.

6. Ito T, Ikeda S, Asamori T, Honda K, Kawashima Y, Kitamura K, et al. Increased expression of pendrin in eosinophilic chronic rhinosinusitis with nasal polyps. Braz J Otorhinolaryngol.2019;85:760-5. 
7. Hu Y, Cao PP, Liang GT, Cui YH, Liu Z. Diagnostic significance of blood eosinophil count in eosinophilic chronic rhinosinusitis with nasal polyps in Chinese adults. Laryngoscope.2012;122:498503.

8. Meng YF, Lou HF, Wang CS, Zhang L. [The value of sinonasal CT scan in diagnosing of eosinophilic chronic rhinosinusitis with nasal polyps]. Zhonghua Er Bi Yan Hou Tou Jing Wai Ke Za Zhi. 2017;52:93-8.

9. Ren L, Zhang L, Duan S, Zhang W, Zhang Y. Nasal airflow resistance measured by rhinomanometry in a healthy population of China. Int Forum Allergy Rhinol. 2018;8:1308-14.

10. Lund VJ, Kennedy DW. Quantification for staging sinusitis. The Staging and Therapy Group. Ann Otol Rhinol Laryngol Suppl. 1995;167:17-21.

11. Lund VJ, Kennedy DW. Staging for rhinosinusitis. Otolaryngol Head Neck Surg. 1997;117:S35-40.12.

12. Orlandi RR, Kingdom TT, Hwang PH, Smith TL, Alt JA, Baroody FM, et al.International Consensus Statement on Allergy and Rhinology: Rhinosinusitis. Int Forum Allergy Rhinol.2016;6 Suppl 1:S22209.

13. Hummel T, Whitcroft KL, Andrews P, Altundag A, Cinghi C, Costanzo RM, et al. Position paper on olfactory dysfunction. Rhinol Suppl. 2017;54:1-30.

14. Mullol J, Marino-Sanchez F, Valls M, Alobid I, Marin C. The sense of smell in chronic rhinosinusitis. J Allergy Clin Immunol. 2020;145:773-6.

15. Haruna S, Otori N, Moriyama H, Nakanishi M. Olfactory dysfunction in sinusitis with infiltration of numerous activated eosinophils. Auris Nasus Larynx. 2006;33:23-30.

16. Rydzewski B, Pruszewicz A, Sulkowski WJ. Assessment of smell and taste in patients with allergic rhinitis. Acta Otolaryngol. 2000;120:323-6.

17. Ferguson BJ. Categorization of eosinophilic chronic rhinosinusitis. Curr Opin Otolaryngol Head Neck Surg. 2004;12:237-42.

18. Fujieda S, Imoto Y, Kato Y, Ninomiya T, Tokunaga T, Tsutsumiuchi T, et al. Eosinophilic chronic rhinosinusitis. Allergol Int. 2019;68:403-12.

19. Castillo Vizuete JA, Sastre J, Del Cuvillo Bernal A, Picado C, Martinez Moragon E, Ignacio Garcia JM, et al. Asthma, rhinitis, and nasal polyp multimorbidities. Arch Bronconeumol.2019;55:146-55.

20. Lou H, Meng Y, Piao Y, Zhang N, Bachert C, Wang C, et al. Cellular phenotyping of chronic rhinosinusitis with nasal polyps. Rhinology. 2016;54:150-9. 
21. Shah SA, Ishinaga $H$, Takeuchi K. Pathogenesis of eosinophilic chronic rhinosinusitis. J Inflamm (Lond). 2016;13:11.

22. Sok JC, Ferguson BJ. Differential diagnosis of eosinophilic chronic rhinosinusitis. Curr Allergy Asthma Rep. 2006;6:203-14.

23. Mullol J, Alobid I, Marino-Sanchez F, Quinto L, de Haro J, Bernal-Sprekelsen M, et al.Furthering the understanding of olfaction, prevalence of loss of smell and risk factors: a population-based survey (OLFACAT study). BMJ Open. 2012;2: e001256.

24. Miwa T, Uramoto N, Tsukatani T, Furukawa M. Middle turbinate fenestration method: a new technique for the treatment of olfactory disturbance due to chronic sinusitis. Chem Senses. 2005;30 Suppl 1:i214-5.

25. Takeno S, Hirakawa K, Ishino T. Pathological mechanisms and clinical features of eosinophilic chronic rhinosinusitis in the Japanese population. Allergol Int. 2010;59:247-56.

26. Vento SI, Simola M, Ertama LO, Malmberg CH. Sense of smell in long-standing nasal polyposis. Am J Rhinol. 2001;15:159-63.

27. Saito T, Tsuzuki K, Yukitatsu Y, Sakagami M. Correlation between olfactory acuity and sinonasal radiological findings in adult patients with chronic rhinosinusitis. Auris Nasus Larynx.2016;43:422-8.

28. Mattos JL. Mechanisms and treatment of olfactory dysfunction in chronic rhinosinusitis. Ann Allergy Asthma Immunol. 2020;124:307-8.

29. Klimek L, Eggers G. Olfactory dysfunction in allergic rhinitis is related to nasal eosinophilic inflammation. J Allergy Clin Immunol. 1997;100:158-64.

30. Aksoy C, Elsurer C, Artac H, Bozkurt MK. Evaluation of olfactory function in children with seasonal allergic rhinitis and its correlation with acoustic rhinometry. Int J Pediatr Otorhinolaryngol. 2018;113:188-91.

31. Mori E, Matsuwaki Y, Mitsuyama C, Okushi T, Nakajima T, Moriyama H. Risk factors for olfactory dysfunction in chronic rhinosinusitis. Auris Nasus Larynx. 2013;40:465-9.

\section{Figures}




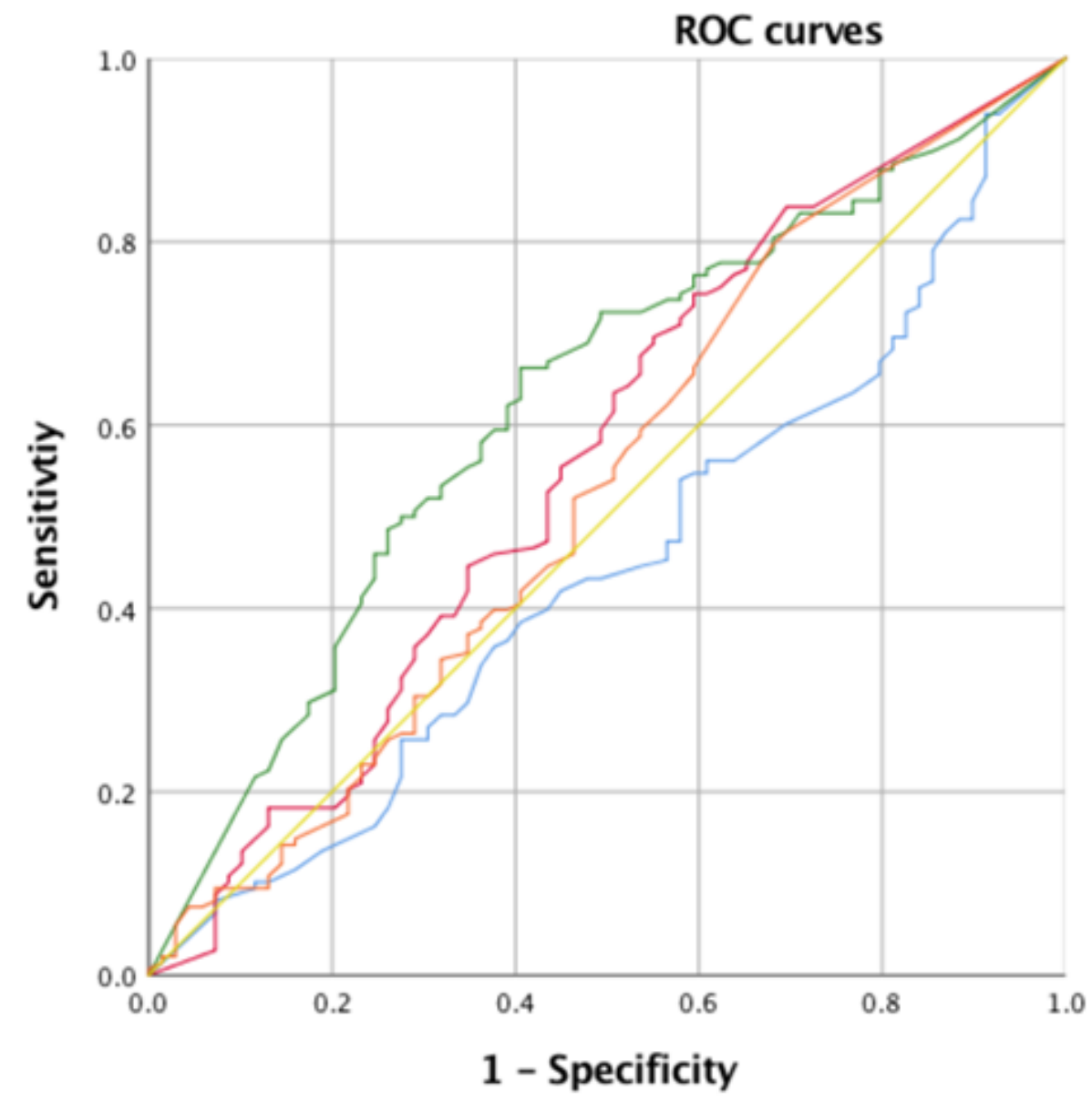

Sources of curves

Nasal congestion

Head/facial pain

Olfactory disorders

Rhinorrhea

Reference

Figure 1

ROC curves of the four symptoms. The AUC of olfactory disorder (AUC $=0.642)$ was higher than those of other symptoms.

\section{Supplementary Files}

This is a list of supplementary files associated with this preprint. Click to download.

- SupplementaryTable1Aug16.docx 